\title{
Nonalcoholic fatty liver disease in type 2 diabetes: awareness is the first step toward change
}

\author{
Fernando Bril ${ }^{1,2}$ \\ ${ }^{1}$ Division of Endocrinology, Diabetes and Metabolism, ${ }^{2}$ Internal Medicine, Department of Medicine, University of Florida, Gainesville, FL, USA \\ Correspondence to: Fernando Bril, MD. Department of Medicine, University of Florida, Gainesville, FL, USA. Email: Fernando.Bril@medicine.ufl.edu. \\ Provenance and Peer Review: This article was commissioned by the editorial office, Hepatobiliary Surgery and Nutrition. The article did not undergo \\ external peer review. \\ Comment on: Younossi ZM, Golabi P, de Avila L, et al. The global epidemiology of NAFLD and NASH in patients with type 2 diabetes: a systematic \\ review and meta-analysis. J Hepatol 2019;71:793-801.
}

Submitted Oct 20, 2019. Accepted for publication Nov 07, 2019.

doi: 10.21037/hbsn.2019.11.11

View this article at: http://dx.doi.org/10.21037/hbsn.2019.11.11

In a recent article published in $\mathcal{F}$ Hepatol, Younossi et al. provided a comprehensive systematic review and meta-analysis on the prevalence of NAFLD, NASH and advanced fibrosis in patients with type 2 diabetes mellitus (T2DM) across the globe (1). Among the 80 studies included in the final analysis (for a total of 49,419 patients) the prevalence of NAFLD was between $50-70 \%$ in all continents, except for Africa, where a prevalence of $30.4 \%$ was reported. This lower prevalence of NAFLD in Africa is consistent with prior reports showing a lower prevalence of NAFLD among African-American individuals (2,3). Of note, once African-American patients develop NAFLD, their progression to NASH and advanced fibrosis is similar to other ethnic groups, and still a significant cause of cirrhosis in this population (4).

Surprisingly, Younossi et al. found that $\sim 70 \%$ of patients with T2DM and NAFLD had a diagnosis of NASH, which implied that $\sim 37 \%$ of all patients with T2DM had NASH. Meanwhile, $\sim 17 \%$ of patients with T2DM and NAFLD had advanced fibrosis. However, interpretation of these rates should be done cautiously. While the authors provided a systematic approach and an exhaustive analysis, which strengthened their conclusions, it is undeniable that this article suffers from some limitations, most of which are inherent to the type of study. High heterogeneity among included studies (e.g., including different diagnostic tools to diagnose NAFLD), selection bias of the patients included in the original studies, and referral bias of those patients who underwent a liver biopsy (i.e., highly selected based on a higher risk of NASH or advanced fibrosis) are probably the most significant ones. As a result of these, there is likely an overestimation of the true prevalence of NAFLD, NASH, and advanced fibrosis. Nevertheless, regardless of the actual rates obtained, the most significant contribution of this systematic review is putting in writing something that we were all aware of "NAFLD and NASH are extremely frequent in patients with T2DM, and it is a worldwide epidemic".

To put their results in perspective, we have provided in Figure 1, a summary of the results from this article (1) together with similar results for the overall population presented by the same group (5). Several conclusions can be drawn from this data. First, that liver disease tends to be more common in patients with T2DM, and that these patients usually have more severe forms of the disease. Therefore, healthcare providers should have a low threshold to screen for liver disease in these patients. In line with this, the American Diabetes Association has recently added in their latest guidelines that patients with hepatic steatosis or elevated plasma ALT should be screened for NASH and advanced fibrosis (6). Another important point is that due to the high prevalence of diabetes among patients with severe liver disease, these patients may benefit for diabetes screening (if not previously diagnosed). This includes considering HbA1c or an oral glucose tolerance test in patients with normal fasting plasma glucose.

Younossi et al. were successful in showing us that this is not just a "Western" problem as sometimes it is referred (1). Due to the increasing prevalence of obesity and diabetes worldwide, we are facing a global NAFLD epidemic. 


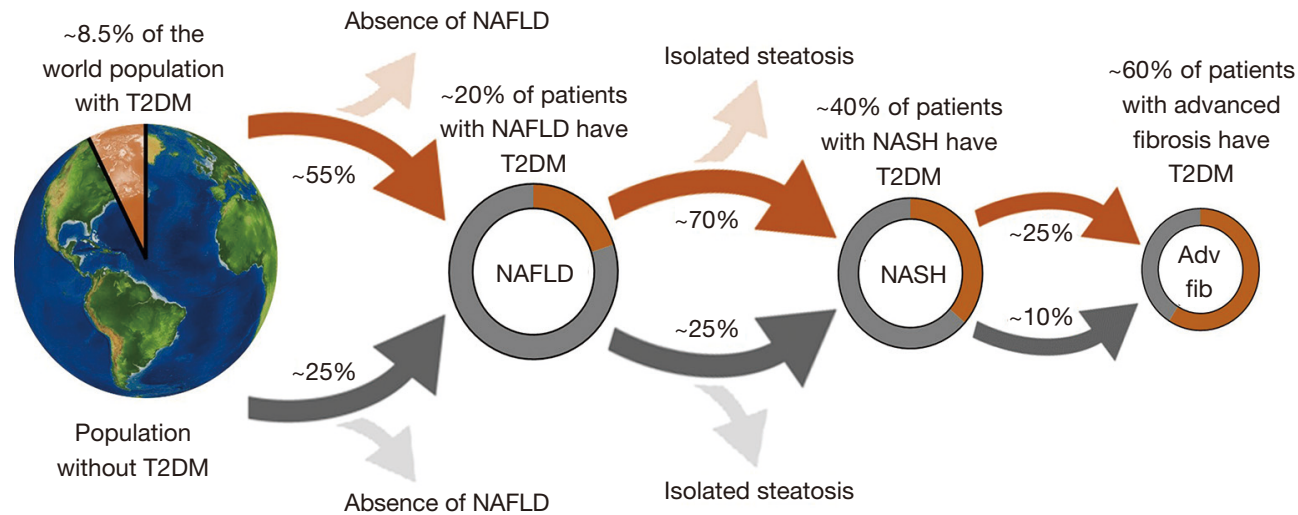

Figure 1 Summary of the prevalence of liver disease among patients with type 2 diabetes mellitus (T2DM) and the overall population based on studies by Younossi et al. $(1,5)$.

Moreover, because of the relatively slow progression of this liver disease, at the present time, we are just seeing the early manifestations of this epidemic. The worst is yet to come, as obese children and young adults continue their growth in a sedentary and nutrient-overloaded environment. Pediatric studies have shown not only a high prevalence of NAFLD among obese children, but also high progression to NASH and advanced fibrosis $(7,8)$. In this context, Younossi et al.'s article should be conceived as yet another call to action to contain this growing problem. Discussing whether the real worldwide prevalence of NAFLD in T2DM is 50\%, $60 \%$ or $70 \%$ is purposeless. We already have the most accurate estimation: it is way too high and something needs to be done! Similarly, establishing whether the exact prevalence of NASH among patients with T2DM is $20 \%, 25 \%$, or $35 \%$ should not be the ultimate goal. The meaning is the same: $\mathrm{NASH}$ is highly prevalent in this population, and therefore, our efforts should specifically focus on this population.

The article by Younossi et al. also assessed the association between NAFLD and diverse metabolic comorbidities, such as hypertension, dyslipidemia, and atherosclerosis. This supports the current concept that NAFLD is really a systemic disease, characterized by insulin resistance, hyperinsulinemia, and lipotoxicity, which simultaneously affect many organs (9). In this setting, intrahepatic triglyceride accumulation should be understood as a barometer of metabolic health (10). Therefore, it is not surprising that patients with NAFLD and T2DM showed increased overall, cardiovascular and liverrelated mortality in this study (1). Unfortunately, the true nature of the relationship between hepatic and cardiovascular disease in NAFLD is unclear: Is one responsible for the other or are both the consequence of an underlying factor?
Due to the diversity of coexisting metabolic factors in these patients, answering this question may prove to be harder than expected.

The current dogma among hepatologists is that liver fibrosis is the strongest predictor of outcomes in patients with NAFLD. This is supported by studies that found higher overall as well as liver-related mortality with increasing fibrosis stage $(11,12)$. Interestingly, these studies only included patients with a diagnosis of NAFLD, and did not specifically assess cardiovascular mortality. Moreover, the magnitude of increase observed in the overall mortality by fibrosis stage was similar to the increase in liver-related mortality. For example, in the study by Dulai et al. (12), mortality rate (per 1,000 patient-years of follow-up) went from 15.2 (stage 0 ) to 45.8 (stage 4) for overall mortality and from 0.3 to 23.3 for liver-related mortality. This suggests that liver-related mortality was indeed driving the increase in overall mortality. While the relative impact of this is impressive, we need to keep in mind that in absolute terms these patients still primarily die of cardiovascular disease. The available evidence linking liver disease and cardiovascular mortality is rather weak, and always surrounded by a myriad of other metabolic factors acting as confounding factors, especially in patients with T2DM. Targher et al. showed in cross-sectional as well as longitudinal studies that NAFLD was associated with cardiovascular events and mortality $(13,14)$. However, studies comparing isolated steatosis to NASH have not consistently shown increased cardiovascular disease in the latter. Indeed, the majority of these studies showed similar cardiovascular mortality rates among isolated steatosis and NASH (15), 


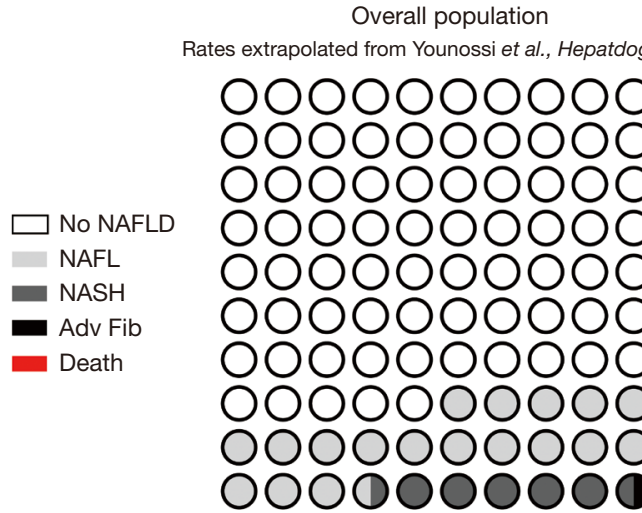

Rates extrapolated from Younossi et al. Hepatdogy 2016

Patients with T2DM

Rates extrapolated from Younossi et al., Hepatdogy 2019.

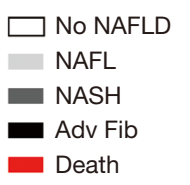

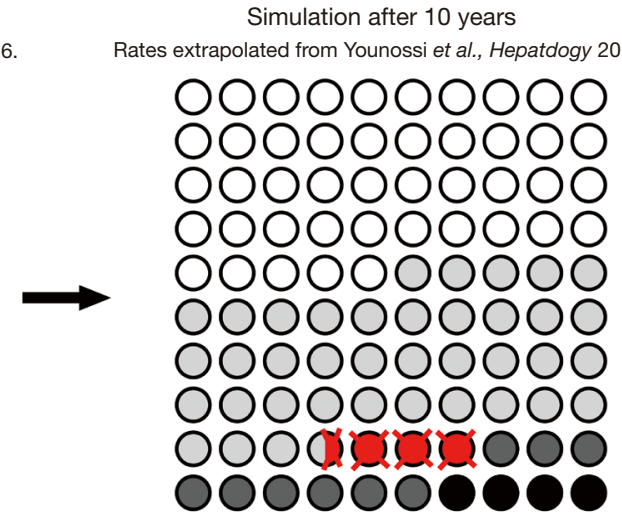

Simulation after 10 years

Rates extrapolated assuming RR $\sim 1.5$ for patients with T2DM

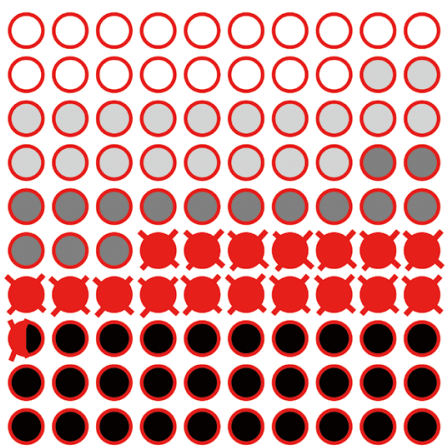

Figure 2 Prediction of progression of liver disease and mortality after 10 years of follow-up in patients with type 2 diabetes mellitus (T2DM) and the overall population based on results from prior studies $(1,5)$.

suggesting a similarly increased risk among all NAFLD patients. Moreover, patients with stage 3 fibrosis had more vascular events than patients with cirrhosis (stage 4) in the study by Vilar-Gomez et al. (16), implying that fibrosis stage may not be the primary determinant of the rate of cardiovascular events.

In Figure 2, we have attempted to summarize the available evidence provided by Younossi et al.'s manuscript in patients with T2DM in combination with prior studies carried out in the overall population. We then extrapolated based on available rates, the fate of 100 patients with and without diabetes after 10 years. While these are only approximate estimates, they provide an idea of the magnitude of the problem. As can be observed in the figure, not only this is a highly prevalent problem, but also it is a progressive problem, with an unrelenting growth, and significant morbidity and mortality. In summary, while liverrelated complications are important, especially in relative terms, these patients are mainly dying of cardiovascular complications.

Dr. Younossi and collaborators should be congratulated for presenting a comprehensive analysis of the available evidence, which I hope will help opening our eyes to the enormous problem that lays in front of us. But awareness is just the first step. In order to appropriately tackle this public health problem, we need to complement it with good non-invasive diagnostic tools and safe and effective therapeutic options, which are unfortunately still lacking. Until these become available, it will be difficult to achieve a major paradigm shift among healthcare providers, as they frequently stay away from ordering a liver biopsy due to the misconception that "there is nothing to offer to patients with NAFLD". However, in the last few years, several non-invasive tests to diagnose NAFLD and NASH, as well as promising treatments have been described $(9,15)$. Hopefully, we will soon count with all the suitable tools to fight this epidemic. 


\section{Acknowledgments}

Funding: None.

\section{Footnote}

Conflicts of Interest: The author has completed the ICMJE uniform disclosure form (available at http://dx.doi. org/10.21037/hbsn.2019.11.11). The author has no conflicts of interest to declare.

Ethical Statement: The author is accountable for all aspects of the work in ensuring that questions related to the accuracy or integrity of any part of the work are appropriately investigated and resolved.

Open Access Statement: This is an Open Access article distributed in accordance with the Creative Commons Attribution-NonCommercial-NoDerivs 4.0 International License (CC BY-NC-ND 4.0), which permits the noncommercial replication and distribution of the article with the strict proviso that no changes or edits are made and the original work is properly cited (including links to both the formal publication through the relevant DOI and the license). See: https://creativecommons.org/licenses/by-nc-nd/4.0/.

\section{References}

1. Younossi ZM, Golabi P, de Avila L, et al. The global epidemiology of NAFLD and NASH in patients with type 2 diabetes: A systematic review and meta-analysis. J Hepatol 2019;71:793-801.

2. Bril F, Portillo-Sanchez P, Liu IC, et al. Clinical and histologic characterization of nonalcoholic steatohepatitis in African American patients. Diabetes Care 2018;41:187-92.

3. Browning JD, Szczepaniak LS, Dobbins R, et al. Prevalence of hepatic steatosis in an urban population in the United States: impact of ethnicity. Hepatology 2004:40:1387-95.

4. Setiawan VW, Stram DO, Porcel J, et al. Prevalence of chronic liver disease and cirrhosis by underlying cause in understudied ethnic groups: The multiethnic cohort. Hepatology 2016;64:1969-77.

5. Younossi ZM, Koenig AB, Abdelatif D, et al. Global epidemiology of nonalcoholic fatty liver disease-Metaanalytic assessment of prevalence, incidence, and outcomes. Hepatology 2016;64:73-84.

6. American Diabetes Association. 4. Comprehensive
Medical Evaluation and Assessment of Comorbidities: Standards of Medical Care in Diabetes-2019. Diabetes Care 2019;42:S34-45.

7. Schwimmer JB, Deutsch R, Kahen T, et al. Prevalence of fatty liver in children and adolescents. Pediatrics 2006;118:1388-93.

8. Goyal NP, Schwimmer JB. The Progression and Natural History of Pediatric Nonalcoholic Fatty Liver Disease. Clin Liver Dis 2016;20:325-38.

9. Friedman SL, Neuschwander-Tetri BA, Rinella M, et al. Mechanisms of NAFLD development and therapeutic strategies. Nat Med 2018;24:908-22.

10. Bril F, Barb D, Portillo-Sanchez P, et al. Metabolic and histological implications of intrahepatic triglyceride content in nonalcoholic fatty liver disease. Hepatology 2017;65:1132-44.

11. Angulo P, Kleiner DE, Dam-Larsen S, et al. Liver Fibrosis, but No Other Histologic Features, Is Associated With Long-term Outcomes of Patients With Nonalcoholic Fatty Liver Disease. Gastroenterology 2015;149:389-97.e10.

12. Dulai PS, Singh S, Patel J, et al. Increased risk of mortality by fibrosis stage in nonalcoholic fatty liver disease: Systematic review and meta-analysis. Hepatology 2017;65:1557-65.

13. Targher G, Bertolini L, Rodella S, et al. Nonalcoholic fatty liver disease is independently associated with an increased incidence of cardiovascular events in type 2 diabetic patients. Diabetes Care 2007;30:2119-21.

14. Targher G, Bertolini L, Padovani R, et al. Relations between carotid artery wall thickness and liver histology in subjects with nonalcoholic fatty liver disease. Diabetes Care 2006;29:1325-30.

15. Bril F, Cusi K. Management of nonalcoholic fatty liver disease in patients with type 2 diabetes: A call to action. Diabetes Care 2017;40:419-30.

16. Vilar-Gomez E, Calzadilla-Bertot L, Wai-Sun Wong V, et al. Fibrosis Severity as a Determinant of Cause-Specific Mortality in Patients With Advanced Nonalcoholic Fatty Liver Disease: A Multi-National Cohort Study. Gastroenterology 2018;155:443-57.e17.

Cite this article as: Bril F. Nonalcoholic fatty liver disease in type 2 diabetes: awareness is the first step toward change. HepatoBiliary Surg Nutr 2020;9(4):493-496. doi: 10.21037/ hbsn.2019.11.11 